\title{
Osteoporosis in Male Ankylosing Spondylitis Patients
}

Research Article

Soliman E

Department of Internal Medicine, Alexandria University, Egypt.

\section{Introduction}

Ankylosing Spondylitis (AS) is a chronic disease with inflammation at the enthesis of the spine and peripheral skeleton, which leads to bony erosion or juxtain sertional osteoporosis in the early stages. However, in later stages, new bone formation, syndesmophytes and ankylosis can occur. Generalized as well as regional osteopaenia is common in AS [1] with a frequency ranging from $50-92 \%$ [2-4]. It ranges from $41-62 \%$ in spine and $46-86 \%$ in femur [5].

Osteoporosis in AS is multi-factorial. It could be due to steroids, prolonged use of non-steroidal anti inflammatory drugs (NSAIDs), decreased mobility, a deficit in sex hormone secretion, or even the inflammatory process. Another possible factor is mineralization defects caused by impaired calcium and vitamin D absorption due to subclinical gut involvement. Recently, the possible role of inflammatory activity has been proposed in several diseases $[1,6]$.

Inflammatory activity in AS plays a major role in the pathophysiology of bone loss, this is mediated by cytokines regulating both the inflammatory process and bone resorption. When assessing patients with AS, it is useful to measure disease activity which reflects acute inflammation, physical function reflects the impact the disease has on the patient's ability to perform activities in daily life and structural damage which reflects the end result of the AS process on anatomical structures [7].

In AS two enhanced but opposite bone remodelling processes take place within the spine which are pathologic new bone formation in the cortical zone of the vertebrae, the zygapophyseal joints, and the ligamentous apparatus and excessive loss of trabecular bone in the centre of the vertebral body. This leads to osteoporosis early in the disease $\&$ increased bone mass later due to syndesmophyte formation [6]. These syndesmophytes result in false increase in bone mineral density (BMD) as assessed by conventional postero-anterior dual energy X-ray absorptiometry (DEXA). Currently, femoral DEXA is thought to be the most accurate method for diagnosis of osteoporosis in AS, but we believe that this is applicable only to advanced AS. On the other hand, lumbar spine is a more sensitive site for the detection of osteopenia than the proximal femur in patients with mild AS [2].

The aim of this study was to assess osteoporosis in male AS patients, its relation to the activity, severity and duration of the disease.

\section{Patients and Methods}

\section{Patients}

Twenty male patients fulfilled the 1984 modified New York criteria for AS were included in the study [8]. They were selected from outpatient clinic of internal medicine department.

Those with risk factors of osteoporosis were excluded e.g. drug intake known to affect bone metabolism (steroids or anticonvulsants), excessive alcohol intake, endocrine disorders (thyroid \& parathyroid), chronic renal \& liver diseases, inflammatory bowel disease, psoriasis or metabolic diseases (Diabetes).

The study was approved by the local ethics committee and was performedin accordance with ethical standards of the 1964 Declarationof Helsinki. Patients gave informed consent to be included in the study.

\section{Methods}

Detailed history \& physical examination of the axial skeleton, peripheral joints and enthesis were done. Also occiput to wall, chest expansion and modified schober tests were done. Measurement of Bath Ankylosing Spondylitis Disease Activity Index (BASDAI) [9], Bath Ankylosing Spondylitis Functional Index (BASFI) [10],

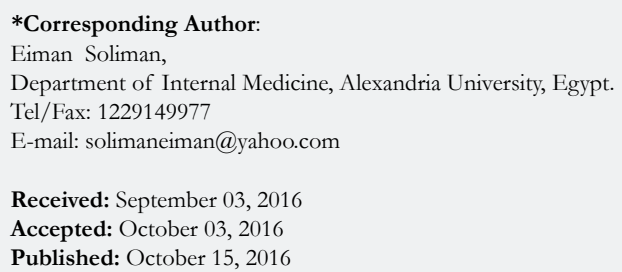


and Bath Ankylosing Spondylitis Metrology Index (BASMI) [11] were carried out.

Bone mass was measured at the femur and lumbar spine (L2-L4) (AP and lateral) using DEXA (HOLOGIC quantitative digital radiography (QDR-4500W). Results were expressed as BMD (gm/ $\mathrm{cm}^{2}$ ). BMD was defined according to the established criteria of the World Health Organization (WHO), which is based on data of postmenopausal women as no criteria of osteopenia and osteoporosis are available for males. T-scores (number of standard deviations (SD) from the normal mean obtained from young healthy people $=$ peak bone mass) and Z-scores (number of SDs from the normal mean for age and gender). Normal bone density was defined as $\mathrm{T}$ score $\geq-1.0$, osteopenia as $\mathrm{T}$ score between $-1 \&-2.5$, and osteoporosis $\leq-2.5$ [12]. Based on WHO criteria established for osteoporosis in women, osteoporosis was diagnosed in any patient if $\mathrm{T}$ scores $<-2.5 \mathrm{SD}$ in one region was found(lumbar spine or femur). The term spinal DEXA was used collectively for the A-P \& lateral views.

Laboratory investigations including CBC, ESR, CRP, RF, SGOT \& SGPT, blood urea \& serum creatinine as well as HLA-B27 were done

Patients were classified in subgroups: first early or late if disease duration was $<$ or $\geq 10$ years [13]. Second active $\&$ inactive according to BASDAI score $\geq 4$ or less [7]. Third, ESR level $\geq$ $20 \mathrm{mmHg}$ (active) or less (inactive) [13]. Fourth based on modified schober test $\geq 5 \mathrm{~cm}$ (mild) or $<5 \mathrm{~cm}$ (severe) [14] and fifth based on DEXA where we defined normal as T score $>-1$ SD \& "low BMD" as $\leq-1.0 \mathrm{SD}$, including both osteopenia and osteoporosis [15].
Statistical analysis was performed using the Statistical Package for Social Science version 20. Statistical analysis was conducted in four steps. The first step consisted of a description of the clinical, laboratory and BMD data. The second step consisted of comparing patients with early and late disease duration as regards their BMD results. The third step consisted of comparison between the low BMD group and normal group as regards different parameters with special emphasis on disease duration, disease activity and severity (spinal mobility). The differences between the groups were tested for significance using the non-parametric test Mann-Whitney U test for unpaired data. The fourth step consisted of correlations between BMD and different parameters with special emphasis on disease duration, disease activity and severity. Those correlations were presented as spearman's correlation coefficient.

Statistical significance was defined as a $\mathrm{p}<0.05$ for the analysis.

\section{Results}

Twenty male patients with a mean age of $32.35 \pm 12.14$ years were the basis of this study. Their clinical \& laboratory features are presented in Table (1). Peripheral \& axial affection was present in all of our patients. All of them were receiving NSAIDs. The classified subgroups of the included patients are presented in Table 2.

Agreement between the two methods done for evaluation of disease activity was carried out (BASDAI \& ESR) but was not statistically significant.

After DEXA was done six patients had normal BMD \& 8 had low

Table 1. Clinical, Laboratory and Radiologic Features of AS Patients $(n=20)$.

\begin{tabular}{|c|c|c|c|c|c|c|}
\hline Variables & & & Mean & Range & Median & SD \\
\hline Age (yr) & & & 32.35 & $18-59$ & 30.5 & 12.14 \\
\hline Age at disease onset (yr) & & & 24.4 & $14-40$ & 22.5 & 7.63 \\
\hline Disease duration (yr) & & & 8.3 & $1-21$ & 7 & 6.05 \\
\hline Occiput to wall $(\mathrm{cm})$ & & & 22.85 & $11.5-32$ & 25 & 6.19 \\
\hline Chest expansion $(\mathrm{cm})$ & & & 2.58 & $0-6.5$ & 2.25 & 1.52 \\
\hline Modified Schobar test $(\mathrm{cm})$ & & & 3.9 & $1.5-6.5$ & 3.5 & 1.27 \\
\hline BASDAI & & & 5.5 & $2.1-8.6$ & 5.65 & 2.15 \\
\hline BASFI & & & 5.4 & $1-9.1$ & 5.7 & 2.58 \\
\hline BASMI & & & 1.63 & $0.4-4$ & 1.4 & 1.18 \\
\hline $\mathrm{ESR}(\mathrm{mm} / \mathrm{hr})$ & & & 39.25 & $5-90$ & 37.5 & 24.04 \\
\hline $\mathrm{BMD}\left(\mathrm{gm} / \mathrm{cm}^{2}\right)$ & Femur & & 0.94 & $0.7-1.18$ & 0.92 & 0.16 \\
\hline & Spine (AP) & & 0.96 & $0.74-1.3$ & 0.93 & 0.18 \\
\hline & Spine (lateral) & & 0.94 & $0.71-1.33$ & 0.93 & 0.19 \\
\hline DEXA WHO classification & \multirow{2}{*}{ Femur } & T-score & -0.82 & $-2.7-0.9$ & -0.85 & 1.13 \\
\hline & & Z-score & -0.7 & $-2.4-1$ & -0.8 & 1.16 \\
\hline & \multirow{2}{*}{ Spine AP } & T-score & -1.23 & $-3.3-1.9$ & -1.5 & 1.62 \\
\hline & & Z-score & -1.02 & $-3.3-2.3$ & -1.5 & 1.8 \\
\hline & Spine lateral & T-score & -1.39 & $-3.4-2.2$ & -1.5 & 1.68 \\
\hline
\end{tabular}

AS: Ankylosing Spondylitis, BASDAI: Bath Ankylosing Spondylitis Diseaseactivity Index, BASFI: Bath Ankylosing Spondylitis Functional index, ESR: Erythrocyte Sedimentation Rate. 
Table 2. Frequency of Status of Disease Activity, Severity, Disease Duration \& WHO DEXA Classification among the Studied AS Population.

\begin{tabular}{|c|c|c|c|c|}
\hline \multicolumn{2}{|l|}{ Variables } & & Frequency & $\%$ \\
\hline \multirow{2}{*}{\multicolumn{2}{|c|}{ Disease duration }} & $<10$ yrs = Early & 11 & 55 \\
\hline & & $\geq 10$ yrs $=$ Late & 9 & 45 \\
\hline \multirow{2}{*}{\multicolumn{2}{|c|}{ ESR based disease activity }} & Inactive $=<20$ & 5 & 25 \\
\hline & & Active $=\geq 20$ & 15 & 75 \\
\hline \multirow{2}{*}{\multicolumn{2}{|c|}{ BASDAI based disease activity }} & Inactive $=<4$ & 5 & 25 \\
\hline & & Active $=\geq 4$ & 15 & 75 \\
\hline \multirow{2}{*}{\multicolumn{2}{|c|}{ Modified Schobarbased disease severity }} & Mild $\geq 5 \mathrm{~cm}$ & 4 & 20 \\
\hline & & Severe $<5 \mathrm{~cm}$ & 16 & 80 \\
\hline \multirow{4}{*}{ DEXA WHO classification } & \multirow{2}{*}{ Femoral } & Normal >-1 & 10 & 50 \\
\hline & & Low $\mathrm{BMD} \leq-1$ & 10 & 50 \\
\hline & \multirow{2}{*}{ Spinal } & Normal >-1 & 8 & 40 \\
\hline & & Low $\mathrm{BMD} \leq-1$ & 12 & 60 \\
\hline
\end{tabular}

AS: Ankylosing Spondylitis, BASDAI: Bath Ankylosing Spondylitis Diseaseactivity Index, ESR: Erythrocyte Sedimentation Rate, DEXA: Dual Energy X-ray Absorptiometry.

BMD both spinally \& femorally. Two patients had low femoral BMD with normal spinal BMD \& 4 patients had normal femoral BMD with low spinal BMD. The frequency of patients with normal or low BMD (femoral or spinal) based on T-score is shown in Table (3). Their distribution among the late and the early group is illustrated as well. Spinal osteoporosis was more common than femoral osteoporosis among the early group. As for the late group, femoral osteoporosis was more common than spinal osteoporosis. That is the more time elapses the more spinal BMD increases despite the presence of osteoporosis which makes femoral BMD more reliable for comparisons.

For further study of the effect of disease duration on BMD, the early \& late groups were compared as regards their BMD (Table 4). We found generalized increase in BMD among the late group than in the early group which is more evident as regards the spine and very minimal as regards the hip. This increase was statistically significant for the $\mathrm{Z}$ score of spine (A-P) $(\mathrm{p}=0.05)$, which could be explained by the formation of spinal syndesmophytes which falsely increase the spinal BMD. This supports the superiority of femoral DEXA in assessing osteoporosis especially in late AS.

Finally the disease duration was correlated with BMD (femur \& spine), $T \& Z$ scores of the femur and spine as shown in Table (5). It revealed significant positive correlations of moderate strength for the lumbar spine BMD with disease duration. This indicates that as disease duration increases there is significant increase in BMD of the spine (due to formation of syndesmophytes). As for femoral BMD, there is negative non-significant correlation i.e. its BMD decreases as the disease duration increases but without statistical significance.

Taking the femoral BMD as the reference for subgrouping our patients into normal and low BMD groups, both groups were then compared as regards their clinical criteria to find out the different factors that could affect the development of osteoporosis (Table 6). We found no statistically significant difference between both groups as regards any of the studied parameter, except for the
ESR value reflecting disease activity which is significantly higher among the group with low BMD. Worth noting is the longer disease duration, more limited modified schobar test \& chest expansion \& longer $\mathrm{O}-\mathrm{W}$ distance among the group with low BMD as compared to the group with normal BMD but without statistical significance.

Then the BMD was correlated with the different factors that could possibly affect it (Table 7). The only statistically significant correlation with femoral BMD was the mean ESR value, which was a moderate negative correlation i.e. as ESR increases there is decrease in femoral BMD. As for BASDAI it was a negative correlation as well but not statistically significant. As regards spinal mobility it was a direct correlation but not statistically significant i.e. decrease in spinal mobility corresponds to decrease in BMD.

\section{Discussion}

AS it is a chronic inflammatory disease characterised by bone loss with subsequent osteoporosis as well as osteoproliferation with the development of syndesmophytes. Chronic inflammation and inflammatory mediators provoke osteoporosis which increases the risk of fractures despite the presence of syndesmophytes [16].

The prevalence of low BMD in the current study was 12 \& 10 patients in the spine $\&$ femur respectively (i.e. $60 \& 50 \%$ ). These data are consistent with previous reported prevalence of osteopenia or osteoporosis in AS patients that vary from 50-92\% [2-4]. Since distribution of osteoporosis differs according to disease chronicity, we compared its distribution (femoral and spinal) among the early then among the late group. It was found that among the early group the frequency of spinal osteoporosis was higher than the femoral $(72.7 \%$ verus $45.5 \%$ respectively). On the other hand, among the late group femoral osteoporosis was more frequent than spinal (55.6\% versus $44.4 \%$ respectively). Patients with early and late groups were then compared as regards the mean BMD and mean $\mathrm{T} \& \mathrm{Z}$ scores at both the femur and spine. There was increase in BMD among the late group than in the early group 
Table 3. Frequency of Normal and Low BMD Patients Based on Femoral or Spinal DEXA among the Early and Late Group of Patients.

\begin{tabular}{|c|c|c|c|c|c|c|}
\hline & \multicolumn{2}{|c|}{ Early group } & \multicolumn{2}{c|}{ Late group } & \multicolumn{2}{c|}{ Total } \\
\hline & Femur & Spine & Femur & Spine & Femur & Spine \\
\hline Normal & $6(54.5 \%)$ & $3(27.3 \%)$ & $4(44.4 \%)$ & $5(55.6 \%)$ & 10 & 8 \\
\hline Low BMD & $5(45.5 \%)$ & $8(72.7 \%)$ & $5(55.6 \%)$ & $4(44.4 \%)$ & 10 & 12 \\
\hline Total & 11 & 11 & 9 & 9 & 20 & 20 \\
\hline
\end{tabular}

DEXA: Dual Energy X-ray Absorptiometry, BMD: Bone Mineral Density.

Table 4. Comparison between Early and Late Disease Duration Based on BMD and WHO Classification for DEXA.

\begin{tabular}{|c|c|c|c|c|c|}
\hline \multirow{2}{*}{\multicolumn{3}{|c|}{ DEXA }} & \multicolumn{2}{|c|}{ Disease duration } & \multirow{3}{*}{$\begin{array}{c}\mathbf{p} \\
0.93\end{array}$} \\
\hline & & & \multirow{2}{*}{$\begin{array}{c}\begin{array}{c}\text { Early }(<\mathbf{1 0 y r s}) \\
\text { No=11 }\end{array} \\
-0.84 \pm 1.18 \\
\end{array}$} & \multirow{2}{*}{$\begin{array}{c}\text { Late }(\geq 10 \text { yrs }) \\
\text { No=9 } \\
-0.8 \pm 1.14\end{array}$} & \\
\hline \multirow{5}{*}{ WHO classification } & \multirow{2}{*}{ Hip } & T score & & & \\
\hline & & Z score & $-0.76 \pm 1.13$ & $-0.61 \pm 1.14$ & 0.85 \\
\hline & \multirow{2}{*}{ Spine (AP) } & T score & $-1.77 \pm 1.32$ & $-0.57 \pm 1.79$ & 0.13 \\
\hline & & Z score & $-1.76 \pm 1.33$ & $-0.11 \pm 1.96$ & 0.05 \\
\hline & Spine (lateral) & T score & $-1.93 \pm 1.33$ & $-0.72 \pm 1.9$ & 0.13 \\
\hline \multirow{3}{*}{$\mathrm{BMD}\left(\mathrm{gm} / \mathrm{m}^{2}\right)$} & \multicolumn{2}{|l|}{ Hip } & $0.92 \pm 0.17$ & $0.95 \pm 0.14$ & 0.68 \\
\hline & \multicolumn{2}{|c|}{ Spine (AP) } & $0.9 \pm 0.15$ & $1.03 \pm 0.2$ & 0.12 \\
\hline & \multicolumn{2}{|c|}{ Spine (lateral) } & $0.87 \pm 0.15$ & $1.01 \pm 0.21$ & 0.1 \\
\hline
\end{tabular}

WHO: World Health Organisation, BMD: Bone Mineral Density, DEXA: Dual Energy X-ray Absorptiometry.

Table 5. Correlation Coefficients (r) for Disease Duration and Regional bone Mineral Density in AS Patients ( $\mathrm{n}=20)$.

\begin{tabular}{|c|c|c|c|}
\hline \multirow{2}{*}{\multicolumn{2}{|c|}{ Region }} & \multicolumn{2}{|c|}{ Disease duration } \\
\hline & & \multirow{2}{*}{$\begin{array}{c}\mathbf{R}^{*} \\
-0.06 \\
\end{array}$} & \multirow{2}{*}{$\begin{array}{c}\text { P value } \\
0.79 \\
\end{array}$} \\
\hline \multirow{3}{*}{ Femur } & T score & & \\
\hline & Z score & -0.02 & 0.94 \\
\hline & BMD & -0.006 & 0.98 \\
\hline \multirow{3}{*}{ Spine (A-P) } & T score & 0.46 & 0.04 \\
\hline & Z score & 0.59 & 0.006 \\
\hline & $\mathrm{BMD}$ & 0.46 & 0.04 \\
\hline \multirow{2}{*}{ Spine (lateral) } & T score & 0.46 & 0.04 \\
\hline & BMD & 0.47 & 0.04 \\
\hline
\end{tabular}

*Spearman Correlation.

which is more evident as regards the spine (A-P \& lateral) and very minimal at the hip. This was statistically significant for the spinal DEXA (A-P). This could be explained by the presence of paravertebral bone and ligamentous calcification and ossification (syndesmophytes) in patients with chronic disease. Finally the BMD as well as $\mathrm{T} \& \mathrm{Z}$ scores were correlated with disease duration. A significant positive correlationof moderate strength between the spine BMD \& disease duration was found \& a negative correlation between disease duration and BMD at the femur. That is as the disease duration increases there is corresponding statistically significant increase in BMD of the spine \& a decrease in BMD of the femur but without statistical significance. These syndesmophytes falsely increase the lumbar spine BMD, therefore decreasing the sensitivity of spinal bone densitometry in patients with longstanding disease [2]. This fact could explain why Reid et al., [17], Donnelly et al., [18] \& Mullaji and Upadhyay [19] found a significant increase in lumbar spine BMD values in their patients with long-standing disease. On the other hand they found significant decrease in lumbar spine BMD in AS populations with shorter disease duration. This has been shown by Donnelly at al. when studying femoral neck bone over time in AS population [18]. Since $45 \%$ of our cases had AS for $>10$ years, so the spinal BMD wouldn't be accurate for assessing osteoporosis. This has been concluded by other investigators as well [16]. Hence femoral $\mathrm{BMD}$ was used for the comparisons.

To study the different factors that could affect the development of osteoporosis, patients were divided into 2 groups based on 
Table 6. Comparison between the Normal and Low BMD Groups as Regards Clinical and Laboratory Data.

\begin{tabular}{|c|c|c|c|c|c|c|}
\hline \multirow{2}{*}{ Variable } & \multicolumn{5}{|c|}{ Femoral DEXA } & \multirow{2}{*}{ Z } \\
\cline { 2 - 3 } & \multicolumn{2}{|c|}{ T score } & \multicolumn{2}{|c|}{ Z score } \\
\cline { 2 - 3 } & Normal (10) & $\begin{array}{c}\text { Low BMD } \\
\mathbf{( 1 0 )}\end{array}$ & & $\begin{array}{c}\text { Normal } \\
\mathbf{( 1 2 )}\end{array}$ & $\begin{array}{c}\text { Low BMD } \\
\mathbf{( 8 )}\end{array}$ & P \\
\hline Disease duration & $7.6 \pm 4.38$ & $9 \pm 7.56$ & 0.62 & $7.58 \pm 4.83$ & $9.38 \pm 7.78$ & 0.53 \\
\hline BASDAI & $5.67 \pm 2.02$ & $5.33 \pm 2.37$ & 0.73 & $5.59 \pm 1.56$ & $5.29 \pm 2.99$ & 0.38 \\
\hline BASFI & $5.24 \pm 2.45$ & $5.56 \pm 2.82$ & 0.79 & $5.48 \pm 2.4$ & $5.29 \pm 2.99$ & 0.38 \\
\hline BASMI & $1.78 \pm 1.38$ & $1.47 \pm 0.1$ & 0.57 & $1.68 \pm 1.29$ & $1.54 \pm 1.07$ & 0.76 \\
\hline O-W & $21.7 \pm 6.15$ & $24 \pm 6.32$ & 0.42 & $22.67 \pm 6.1$ & $23.13 \pm 6.73$ & 0.88 \\
\hline Chest expansion & $2.95 \pm 1.62$ & $2.2 \pm 1.38$ & 0.28 & $2.92 \pm 1.49$ & $2.06 \pm 1.52$ & 0.23 \\
\hline Modified Schobar & $4.05 \pm 1.12$ & $3.75 \pm 1.46$ & 0.61 & $4 \pm 1.02$ & $3.75 \pm 1.65$ & 0.68 \\
\hline ESR & $31 \pm 18.43$ & $47.5 \pm 27$ & 0.12 & $28.33 \pm 18.3$ & $55.6 \pm 23.05$ & 0.009 \\
\hline
\end{tabular}

BASDAI: Bath Ankylosing Spondylitis Disease activity Index, BASFI: Bath Ankylosing Spondylitis Functional index, BASMI: Bath Ankylosing Spondylitis Metrology Index, ESR: Erythrocyte Sedimentation Rate, BMD: Bone Mineral Density.

Table 7. Correlation between Femoral BMD and Different Clinical Variables.

\begin{tabular}{|c|c|c|}
\hline \multirow{2}{*}{} & \multicolumn{2}{|c|}{ Femoral BMD } \\
\cline { 2 - 3 } & $\mathbf{r}^{*}$ & $\mathbf{p}$ \\
\hline ESR & -0.53 & 0.02 \\
\hline BASDAI & -0.02 & 0.94 \\
\hline BASFI & -0.16 & 0.5 \\
\hline BASMI & 0.03 & 0.89 \\
\hline Modified Schobar & 0.18 & 0.46 \\
\hline
\end{tabular}

BASDAI: Bath Ankylosing Spondylitis Disease activity Index, BASFI: Bath Ankylosing Spondylitis Functional Index, BASMI: Bath Ankylosing Spondylitis Metrology Index, ESR: Erythrocyte Sedimentation Rate, BMD: Bone Mineral Density *Spearman Correlation.

femoral BMD: normal and low BMD (including osteoporosis \& osteopenia). There was no statistically significant difference between both groups as regards any parameter except for ESR mean value which was significantly higher in the group of low BMD. Worth noting is the more limited modified Schober test \& chest expansion \& longer O-W distance as well as longer disease duration in the group with low BMD than the normal group but without statistical significance.

Disease activity has been assessed in this study using BASDAI \& ESR. Both of which have shown no mutual statistically significant agreement. This lack of a comprehensive relationship between acute-phase reactants \& clinical variables $\&$ imaging with disease process has been highlighted by other investigators. Evaluation of disease activity in AS is very difficult with no gold standard due to wide spectrum of clinical presentation [20].

Regarding disease activity as measured by BASDAI its mean value was higher in the osteoporotic group than the normal group taking spinal DEXA as a reference (data not shown) (5.93 vs 4.85) but without statistical significance. As for femoral DEXA both groups were statistically similar. Also a negative statistically insignificant correlation between BASDAI \& BMD was found. Although BASDAI is a widely used tool for measuring disease activity which has proven validity, reproducibility \& response to change but it is still a subjective method [20].
ESR has been suggested to have limited value in determining disease activity, but it was shown to change in the presence of peripheral joint involvement with respect to patients with pure axial disease [20]. Since all of our patients had peripheral joint involvement, then ESR measurement would be a reliable indicator of disease activity. It was raised above $20 \mathrm{mmHg}$ in 15 of our patients (75\%) which is in accordance with other studies [20]. The mean ESR value was higher among the low BMD group than the normal group when taking the femoral DEXA as the reference with a statistically significant difference when compared with Z score (age matched patients). The effect of ESR on BMD was also emphasized when we correlated the femoral BMD with the different clinical parameters and the only statistically significant correlation was with the mean ESR value which was a negative correlation with a moderate strength. This means that as ESR increases there is decrease in femoral BMD. This is in accordance with researchers who found a clear relationship between loss of bone mass \& laboratory activity as ESR early in the course of the disease [13]. Also a correlation between ESR and bone resorption markers was found in another study by Toussirot et al., [21]. On the other hand other studies investigating this relationship yielded negative results. Inflammatory activity has a significant role in BMD loss in AS. It has been suggested that local or systemic inflammatory cytokine release might be implicated [16]. 
As for modified schober test it was more limited in the group with low BMD than the normal group but without statistical significance. Previous studies support the theory that vertebral restriction isn't an essential factor in early bone loss seen in AS. It might be that spinal limitation as a result of the inflammation itself participates in vertebral bone loss [23, 24].

This study had some limitations such as the small number of patients; however, the study group consisted of drug native patients which made the results highly valuable. Also DEXA measures only bone density which accounts for $70 \%$ of bone strength. However, bone fragility \& consequently fractures depend on bone microarchitecture, which is not assessed by conventional DEXA [22].

\section{Conclusion}

The present study shows not only early bone loss in AS patients with active disease, but also a significant relationship between the inflammation markers as ESR and bone loss. These results clearly support the theory that the inflammatory process plays a pivotal role in the pathophysiology of osteoporosis in AS. Bone loss of vertebral mass occurs early in the course of AS. On the other hand lumbar spine bone densitometry is less sensitive for patients with long-standing AS, due to the false-negative results caused by syndesmophytes. Proximal femur is less affected by diseaserelated new bone formation. Therefore, it should be better used to evaluate bone mass in AS patients with long-standing disease [5]. So bone loss occurs early in the disease and its progression is better assessed by using DXA at the femur.

\section{References}

[1]. Capaci K, hepguler S, Argin M, Tas I (2003) Bone Mineral density in mild and advanced ankylosing spondylitis. Yonsei Med J. 44(3): 379-84.

[2]. Meirelles ES, Borelli A, Camargo OP (1999) Influence of disease activity and chronicity on ankylosing spondylitis bone mass loss. Clin Rheumatol. 18(5): 364-8.

[3]. Devogelaer JP, Maldague B, Malghem J, Nagant de Deuxchaisnes C (1992) Appendicular and vertebral Bone Mass in Ankylosing Spondylitis. A comparison of plain radiographs with single and dual-photon absorptiometry and with quantitative computed tomography. Arthritis Rheum. 35(9): 1062-7.

[4]. El Maghraoul A, Borderie D, Cherruau B, Edouard R, Dougados M, et al., (1999) Osteoporosis, body composition, and bone turnover in ankylosing spondylitis. J Rheumatol. 26(10): 2205-9.

[5]. Kim HR, Kim HY, Lee SH (2006) Elevated levels of soluble receptor activator of nuclear factors $\mathrm{K} \beta$ ligand (sRANKL) and reduced bone mineral density in patients with AS. Rheumatol. 45(10): 1197-2000.

[6]. Sayed S, Darweesh H, Fathy K, Mourad AM (2015) Clinical significance of bone mineral density in Ankylosing Spondylitis patients: Relation to disease activity and physical function. The Egyptian Rheumatologist, 37(1), 35-39.

[7]. Zochling J, Braun J (2005) Assessment of ankylosing spondylitis. Clin Exp Rheumatol. 23(S39): S133-S41.

[8]. Van der Linden S, Valkenburg HA, Cats A (1984) Evaluation of diagnostic criteria for ankylosing spondylitis: a proposal for modification of the New York criteria. Arthritis Rheum. 27(4): 361-8.

[9]. Garrett S, Jenkinson T, Kennedy LG, Whitelock H, Gaisford P, et al., (1994) A new approach to defining disease status in ankylosing spondylitis: The Bath Ankylosing Spondylitis Disease Activity Index (BASDAI). J Rheumatol. 21(12): 2286-91.

[10]. Calin A, Garrett S, Whitelock H, Kennedy LG, O’Hea J, et al., (1994) A new approach to defining functional ability in ankylosing spondylitis: The development of the Bath Ankylosing Spondylitis Functional Index. J Rheumatol. 21(12): 2281-5.

[11]. Jenkinson TR, Mallorie PA, Whitelock HC, Kennedy LG, Garrett SL, et al., (1994) Defining spinal mobility in ankylosing spondylitis (AS). The Bath AS Metrology Index. J Rheumatol. 21(9): 1694-8.

[12]. World Health Organisation (1994) Assessment of fracture risk \& its application to screening for postmenopausal osteoporosis. WHO technical report series 843. Geneva: WHO 1994.

[13]. Grataco's J, Collado A, Pons F, Osaba M, Sanmarti R, et al., (1999) Significant loss of bone mass in patients with early, active ankylosing spondylitis: a followup study. Arthritis Rheum. 42(11): 2319-24.

[14]. Baek HJ, Kang SW, Lee YJ, Shin KC, Lee EB, et al., (2005) Osteopenia in men with mild and severe ankylosing spondylitis. RheumatolInt. 26(1): $30-4$.

[15]. Van der Weijden MAC, Claushuis TAM, Nazari T, Lems WF, Dijkmans BAC, et al., (2012) High prevalence of low bone mineral density in patients within 10 years of onset of ankylosing spondylitis: a systematic review. Clin Rheumatol. 31(11): 1529-35.

[16]. Kaya A, Ozgocmen S, Kamanli A, Ardicoglu O (2009) Bone loss in ankylosing spondylitis: does syndesmophyte formation have an influence on bone density changes?. Med Princ Pract. 18(6): 470-6.

[17]. Reid DM, Nicoll JK, Kennedy NSJ, Smith MA, Tothill P, et al., (1986) Bone mass in ankylosing spondylitis. J Rheumatol. 13(5): 932-935.

[18]. Donnelly S, Doyle DV, Denton A, Rolfe I, McCloskey EV, et al., (1994) Bone mineral density \& vertebral compression fracture rates in ankylosing spondylitis. Ann Rheum Dis. 53(2): 117-21.

[19]. Mullaji AB, Upadhyay SS (1994) Bone mineral density in Ankylosing Spondylitis: DEXA comparison of control subjects with mild \& advanced cases. J Bone Joint Surg [Br]. 76(4): 660-5.

[20]. Ozgocmen S, Godekmerdan A, Ozkurt-Zengin F (2007) Acute phase response, clinical measures $\&$ disease activity in Ankylosing spondylitis. Joint Bone spine. 74(3): 249-53.

[21]. Toussirot E, Ricard-Blum S, Dumoulin G, Cedoz JP, Wendling D (1999) Relationship between urinary pyridinium crosslinks, disease activity and disease subsets of ankylosing spondylitis. Rheumatol. 38(1): 21-7.

[22]. Toussirot E, Michel F, Wendling D (2001) Bone density, ultrasound measurements and body composition in early ankylosing spondylitis. Rheumatol. 40(8): 882-8.

[23]. Lee YS, Schlotzhauer T, Ott SM, van Vollenhoven RF, Hunter J, et al., (1997) Skeletal status of men with early and late Ankylosing spondylitis. Am J Med. 103(3): 233-41.

[24]. Will R, Bhalla AK, Palmer R, Ring R, Calin A (1989) Osteoporosis in early ankylosing spondylitis: a primary pathological event?. Lancet. 2(86788679): 1483-5. 